\title{
Experimental study on counterintuitive behavior of thin aluminium plates under free airblast loading
}

\author{
Zixi Xu, Yan Liu*, and Fenglei Huang \\ State Key Laboratory of Explosion Science and Technology, Beijing Institute of Technology, 100081 Beijing, PR China
}

\begin{abstract}
An experimental investigation of the counterintuitive behaviour in thin aluminium plates under fullfield test is presented in the paper. Special focus is placed on structural response and permanent deflection of the panels after the complex response. Thus, a series of tests with the plates varied in two kinds of thickness were conducted, where impulsive loading was generated by detonating the explosives at various standoff distances. As loaded impulsively, the vibration response was observed and the final configuration of each plate was measured and recreated. It is shown that all plates subjected to free air blast loading in such conditions exhibited plastic permanent deformation combined with distinct counterintuitive behaviour, as the plates deformed to the opposite direction of the incident wave. In addition, the characteristics of the permanent deflection can be divided into 2 typical deformation shapes, and the occurrences of which were correlated to the dimensionless parameter proposed in previous studies, confirmed to be an approximate indicator to describe the influence of loading conditions herein.
\end{abstract}

\section{Introduction}

Since the metal product performs outstanding efficiency on processing and molding, it is extensively employed in constructions and military structures. However, with growing threats from terrorist activities and military actions in recent decades, which will definitely result in unbearable costs and causalities, several efforts have concentrated on improving the mechanics properties of those structures subjected to blast loading [1]. Considering the structural features and material characteristics, the investigations on dynamic behaviour and structural response of aluminium panels subjected to impulsive loading appear to be significant.

However, studies conducted previously were mostly focus on panels made of mild steel, which is a kind of typical rigid-perfectly plastic material, Nurick and Martin $[2,3]$ reported theoretical considerations and experimental studies on blast permanent deformation of thin clamped mild steel plates respectively, where varied boundary conditions were considered. An empirical equation was derived for predicting the trend of the maximum deflection versus the loading impulse through non-dimensional analysis, which was verified to be in great agreement with experimental results in the following investigations on mild steel plates. Afterwards, there was experimental research carried out by Nurick and Shave [4] on the failure modes of strain-sensitive clamped plates as well, three modes (mode I: large ductile deformation, mode II: tensile- tearing at supports and deformation, mode III: transverse shear at supports) were observed, which was in analogy with the definition for those of blast loaded beams [5].

With the conclusions drawn from above, further studies $[6,7]$ focused on structural response in consideration of the effects of structure geometries and material types, which will result in complexity on prediction of blast behaviour and diversity on description of response modes. Likewise, such structural response can be divided into three categories (type I, type II and type III) $[8,9]$, where the phase of type III performs a negative deflection, that is, opposite to the direction of loading. Such a phenomenon named as counterintuitive behaviour (CIB) has drawn much attention in recent decades [10-12].

As a perceivable method to investigate the dynamic response related to blast loading, experimental studies were applied to directly observe the blast behaviour subjected to different conditions and geometries. In aim of comparing the blast behaviour with varied parameters, non-dimensional analysis is extensively utilized for collapsing data and establishing the relationships between the main influencing factors and final damage of the plates. Several non-dimensional numbers for describing loaded impulse were derived $[2,10,13,14]$.

The objective of this study is to investigate the relationship between the concerned non-dimensional loading numbers and dynamic behaviour of clamped aluminium plates under airblast loading. Thus, a series of tests with diverse loading and material parameters were performed in field scale. Considering the damage modes

Corresponding author: liuyan@bit.edu.cn 
and structural response types of the blast-loaded plate, the permanent deflection of panels were chosen for comparing the differences of typical dimensionless numbers. It should be noted that this study has examined only structural response of aluminium plates in large scale.

\section{Experimental setup}

\subsection{Parameters of aluminium plate}

The $2 \mathrm{~mm}$ and $3 \mathrm{~mm}$ thickness plates with an exposed area of $120 \times 100 \mathrm{~cm}^{2}$ were manufactured using aluminium alloy sheets of LY12 according to Chinese standard. The quasistatic material properties of LY12 reported by the producer were applied in this study, no extra property test on LY12 was conducted. Table 1 gives the nominal chemical composition of the material, and a summary of the properties is listed in Table 2, while yield strength and Young's modulus were provided by manufacturer to be $325 \mathrm{MPa}$ and $69 \mathrm{GPa}$.

The frame of the fixture was made of channel steel, which was identical to the material of reinforcement welded internally. In addition, a steel plate of $5 \mathrm{~mm}$ was bolted to the back of the fixture in aim of minimizing the effects caused by diffraction of blast wave.

Table 1. Chemical composition of LY12 (in wt. \%).

\begin{tabular}{ccccc}
\hline $\mathbf{S i}$ & $\mathbf{F e}$ & $\mathbf{C u}$ & $\mathbf{M n}$ & $\mathbf{M g}$ \\
\hline 0.500 & 0.500 & 4.200 & 0.600 & 1.500 \\
\hline $\mathbf{N i}$ & $\mathbf{Z n}$ & $\mathbf{T i}$ & $\mathbf{A l}$ & \\
\hline 0.010 & 0.300 & 0.150 & Rest & \\
\hline
\end{tabular}

Table 2. Material properties.

\begin{tabular}{cccc}
\hline Material & $\begin{array}{c}\text { Density } \\
\boldsymbol{\rho}\left(\mathbf{k g} / \mathbf{m}^{\mathbf{3}}\right)\end{array}$ & $\begin{array}{c}\text { Yield stress } \\
\boldsymbol{\sigma}_{\mathbf{0}}(\mathbf{M P a})\end{array}$ & $\begin{array}{c}\text { Young's } \\
\text { modulus } \\
\boldsymbol{E}(\mathbf{G P a})\end{array}$ \\
\hline $\mathrm{LY} 12$ & 2780 & 325 & 69 \\
\hline
\end{tabular}

The plates were divided into three groups for blast testing and numbered as N-TS, where $\mathrm{N}$ denotes the test number of A, B and C, T is the thickness of the plate and S signifies the serial number of the specimens. Details of specimens are provided in Table 3.

\subsection{Experimental arrangement}

Herein, free airblast loading was generated by detonating the warhead raised off of the floor to the mid-height of the plate. The experimental arrangement is shown in Table 3.
To prevent translation and rotation during blast testing, the fixture was fastened to the floor by steel chisels of $0.5 \mathrm{~m}$. The testing plates of aluminum alloy were bolted to the supporting fixture. Level bar was utilized to keep the plates vertical to the floor during installation. Fig. 1 shows the structure after installation.

Table 3. Details of specimens and experimental setup.

\begin{tabular}{ccccc}
\hline Test & $\boldsymbol{\omega}(\mathbf{k g})$ & ID & $\boldsymbol{h}(\mathbf{m m})$ & $\boldsymbol{r}(\mathbf{m})$ \\
\hline \multirow{3}{*}{ A } & \multirow{3}{*}{1.83} & A-21 & 2 & 10 \\
& & A-22 & 2 & 7 \\
& & A-33 & 3 & 7 \\
& & A-34 & 3 & 7 \\
B & \multirow{3}{*}{16.63} & B-21 & 2 & 15 \\
& & B-22 & 2 & 15 \\
& & B-33 & 3 & 15 \\
C & \multirow{3}{*}{31.61} & C-21 & 2 & 15 \\
& & C-22 & 2 & 15 \\
& & C-33 & 3 & 15 \\
& & C-34 & 3 & 15 \\
\hline
\end{tabular}

Where $\omega$ indicates the equivalent mass of the explosion, $h$ signifies the thickness of the plate and $r$ is the measured standoff distance from location of explosion.

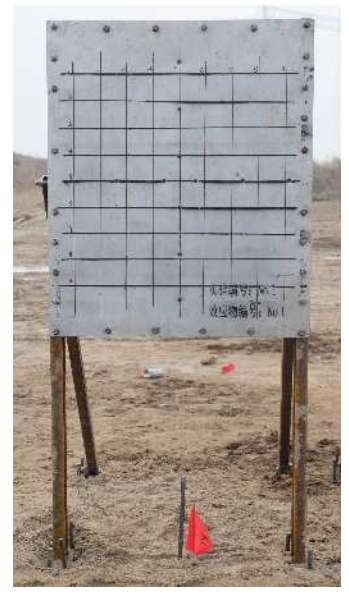

Fig. 1. Photograph of test structure after installation.

As shown in Fig. 1, the front surface of each plate was painted with grid pattern $(100 \times 100 \mathrm{~mm})$ for measuring the final deformation subjected to blast loading, moreover, a right-handed coordinate system was set to facilitate data recording. A device was mounted for the measurement of displacement, as shown in Fig. 2. 


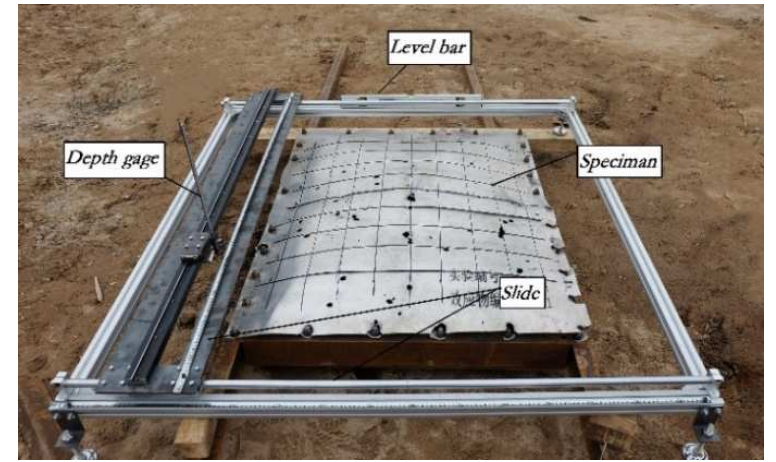

Fig. 2. Photograph of the device for deflection measurement.

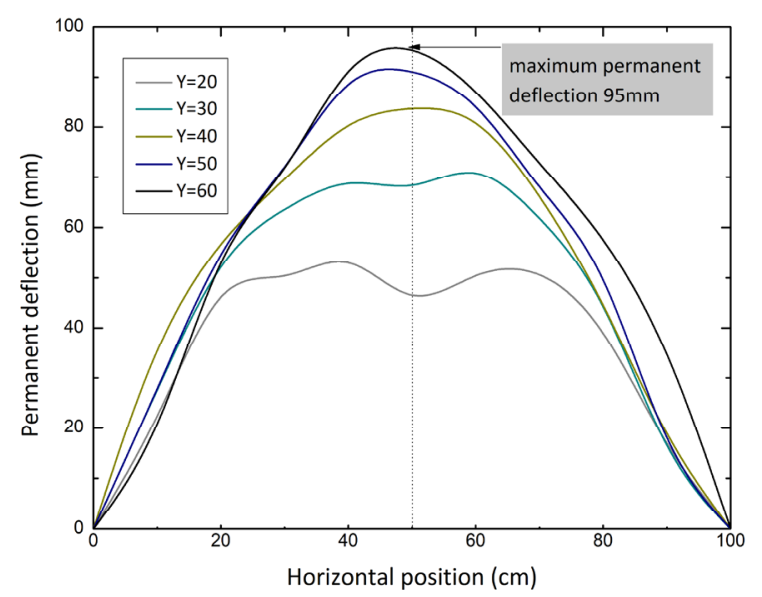

(a)

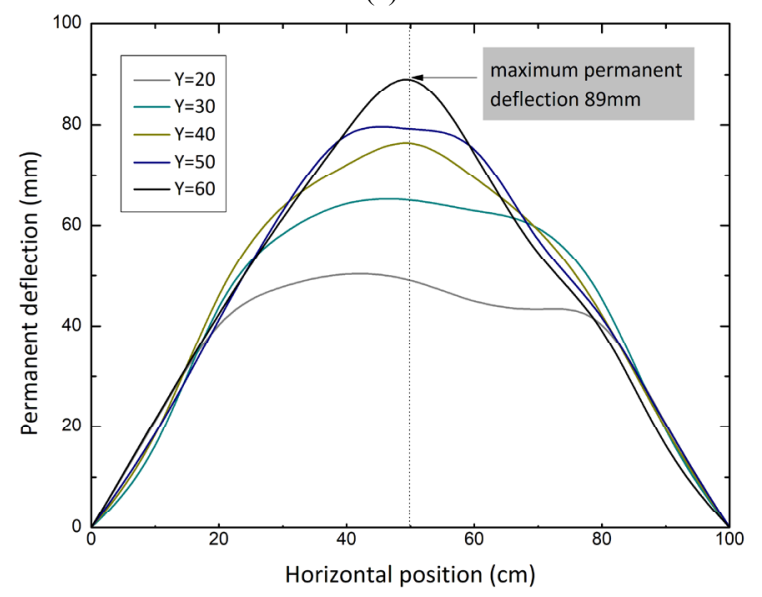

(c)

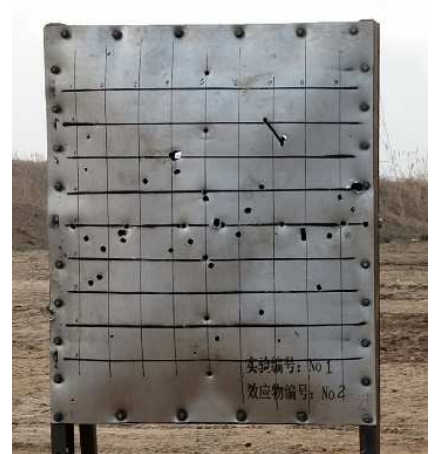

Fig. 3. Photograph of the selected plates (C-34) after test.

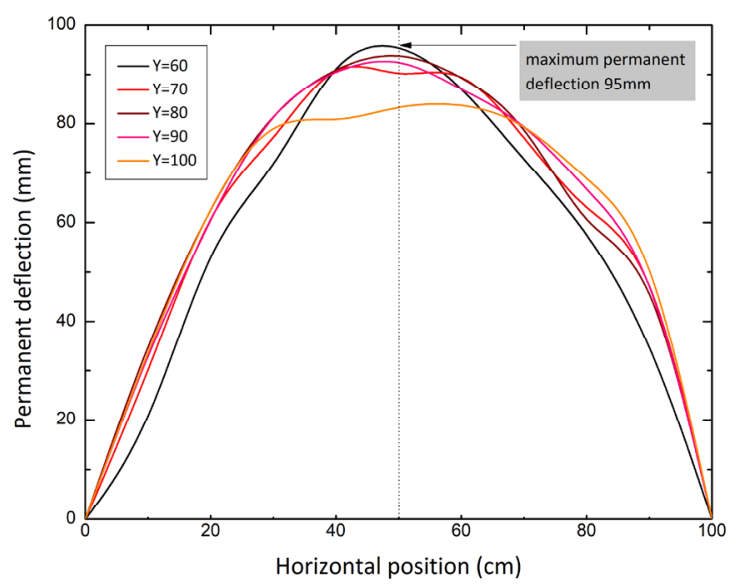

(b)

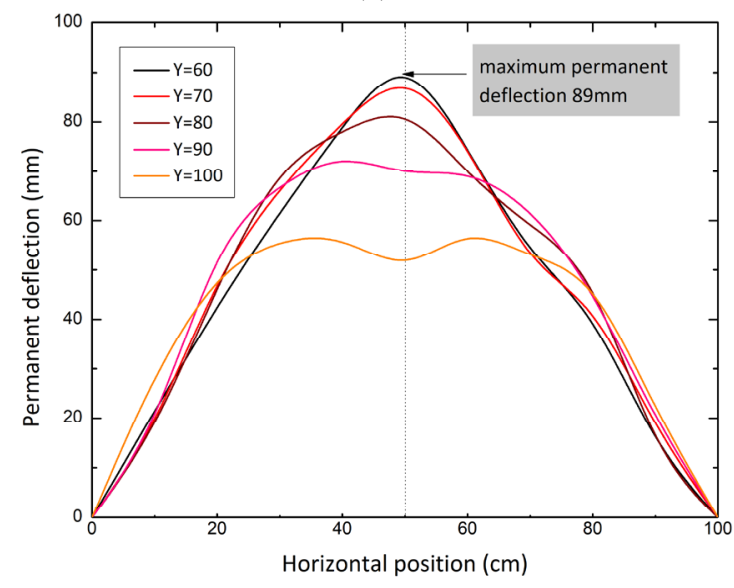

(d)

Fig. 4. Permanent deformation profiles along the horizontal line of the blast loaded plates. (a) $C-33$ : $Y=20-Y=60$; (b) $C-33$ : $Y=60-Y=100$; (c) $\mathrm{C}-34: \mathrm{Y}=20-\mathrm{Y}=60$; (d) $\mathrm{C}-34: \mathrm{Y}=60-\mathrm{Y}=100$.

\section{Performance of the plates}

All the plates deformed to the direction opposite to the impulse loading as shown in Fig. 3, but the performances on counterintuitive behaviour of the plates were diverse. In general, there were two modes observed in the experiments.
As the definition of deformation mode proposed previously [4], the damage of the plates from test-A and test-B and the panels in $3 \mathrm{~mm}$ thickness from test-C can be identified as Mode I. The phenomena should be highlight that the plates of $\mathrm{C}-21$ and $\mathrm{C}-22$ were torn out from the clamping frame along the bottom boundary, which performed a characteristic of Mode II. The results on 
permanent deflection of each plate were recorded making using of the device shown in Fig. 2, except for the slightly deformed plate of A-21.

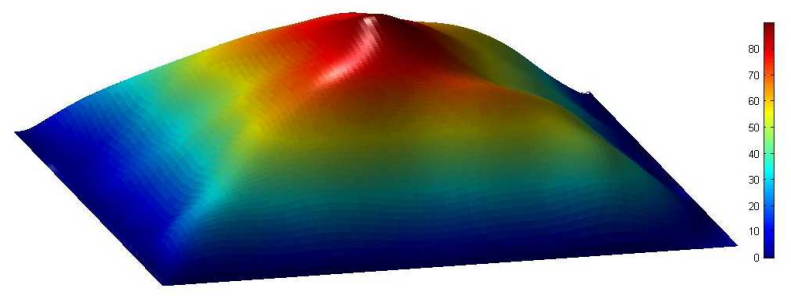

Fig. 5. Result of selected plates (C-34) after surface rendering.

As the plates were painted with grid before, the data were sampled every $10 \mathrm{~cm}$ within the abscissa $X \in(0,100)$ and the ordinate $Y \in(0,120)$. For the better observation on deformed plates, the data recorded from each plate were plotted along the horizontal line of the plate. The plotted curves of each selected plate (C-33 and C-34) provided in Fig. 4 are varied in ordinates and divided into fluctuation two parts for preferably reconstructing the deformation. By comparing the curves in Fig. 4, excellent similarity is found for the damage under the same loading condition.

In addition, since it is difficult to list the deflection of each sampling point from the specimens, the data were imported into MATLAB to make interpolation and surface

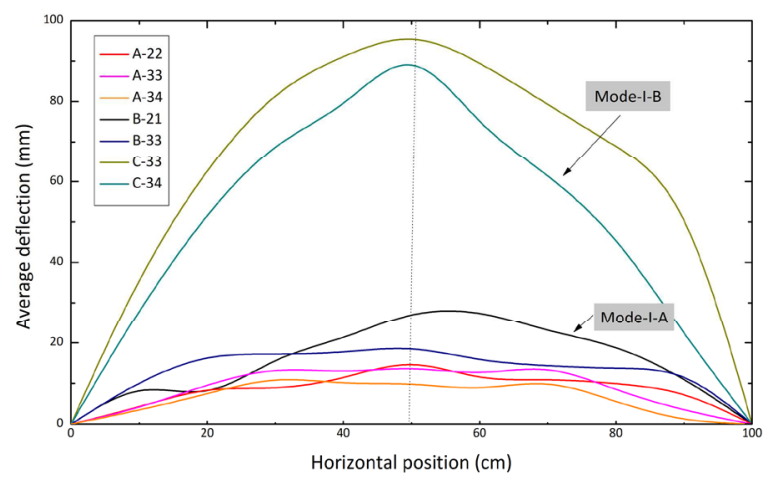

Fig. 6. Maximum permanent deflection along horizontal line of typical plates. rendering. Fig. 5 shows the results of selected plates (C-34) after blast loaded, where plastic hinges along the diagonals of the plates were distinct. It is also observed in the rest plate of test-C, but not visible in other two tests (test-A and test-B) for the little deformation relative to the size of the plates.

As presented in Fig. 6, the maximum permanent deflections from the plates without tearing damage were extracted and summarized to align along horizontal positions and plot together, where the performances of the selected plates appear to be different. The graph revealed that most plates under lower impulse loading in such the scale presented the homogeneous deformation shape with those uniformly loaded in lab scale (defined as Mode I-A in this study). However, as loading impulse increased, the plates deformed similarly to those under localized loading in lab scale, with an obvious peak deflection in the centre of the plates (classified as Mode I-B in this study). To compare directly, typical photographs of lab scale tests were selected from former investigations [4, 15]. Fig. 7 and Fig. 8 present the failure of the plates subjected to uniform and localized loading respectively, where the modes mentioned before could be intuitively observed.

The maximum permanent displacement $\delta_{\max }$ of each plate are listed in Table 4, it was analogously utilized to compare the damage in such conditions, as the mid-point displacement was usually adopted in lab scale tests.

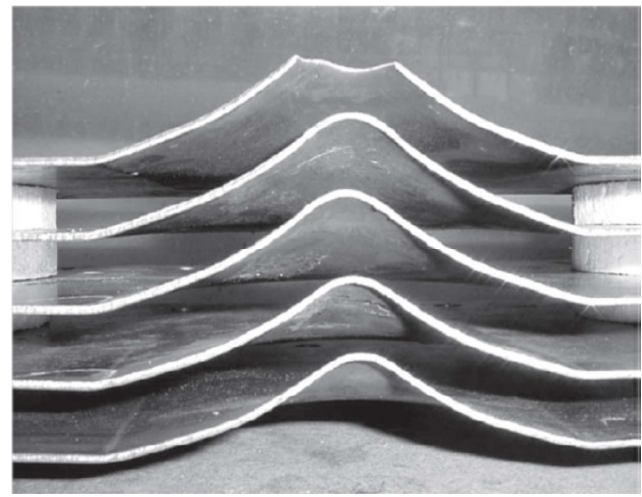

Fig. 7. Photographs of cross-sectional profiles from the plates subjected to localized loading [15].

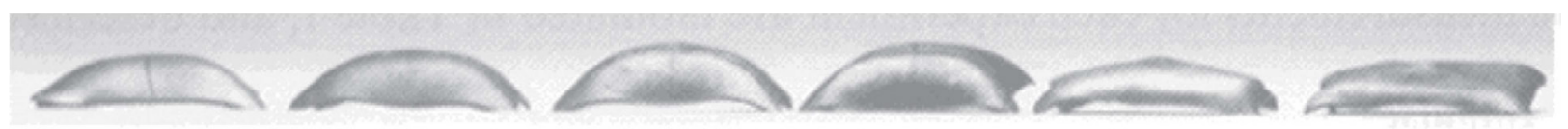

Fig. 8. Photographs showing the failure of uniformly loaded square plates in lab scale [4].

\section{Analysis and comparison}

It can be seen that the blast performance is complicated and sensitive to geometries and loading conditions of the plate, there are even differences in the plates performing the same damage mode. In aim of comparing and distinguishing the blast behaviour with varied parameters, there were several non-dimensional numbers proposed for describing the damage. In consideration of the experimental arrangement set in this paper, two 
dimensionless parameters were employed for further discussion of the observations in the former section.

As advocated by Nurick et al. [2, 3], a non-dimensional impulse parameter for clamped quadrangular plates is shown as below, which is utilized for calculation of the final deflection.

$$
\varphi_{q}=I / 2 h^{2}\left(B L \rho \sigma_{0}\right)^{1 / 2}
$$

Where $I$ - impulse, $h$ - plate thickness, $B$ - plate breadth, $L$ plate length, $\rho$ - material density, $\sigma_{0}$ - quasi-static yield stress. In addition, recent numerical study by E.A. FloresJohnson and Q.M. Li [10] proposed an indicator in aim of investigating the counter-intuitive behavior of aluminium plates, which is as follow, i.e.

$$
\Psi=V_{0} \sqrt{\rho / E}
$$

Where $V_{0}=I / \rho h, E$ - Young's modulus. The parameters of each plate were calculated hereby for further comparison. Details are given in Table 4, where the deformation was adimensionalized as $\Pi_{\max }$, that is, $\Pi_{\max }=\delta_{\max } / h$.

Since all the plate deformed to the negative direction, for such conditions, the counterintuitive behaviour can be always observed in $0.0011<\Psi<0.0053$. Ulteriorly, the phase of mode I appears in condition of $0<\Psi<0.0035$. It seems that $\Psi$ of 0.0034 is a critical value to distinguish the phases of Mode I-A and Mode I-B, but more researches should be conducted to confirm it. For $\varphi_{q}$, it is lack in regularity to describe the permanent damage mode, especially in subdivision of Mode I, but implies to be a significant indicator to predict permanent deflection when the plates deform as Mode I-A.

Considering the limitation of both two parameters, original indicator $\Psi$ integrating the geometries of the plate (breadth and length) will be a novel applicable dimensionless number to estimate the blast behaviour.

Table 4. Summary of deformation and relevant parameters of the plates.

\begin{tabular}{cccccccc}
\hline ID & $\begin{array}{c}\boldsymbol{\delta}_{\max } \\
(\mathbf{m m})\end{array}$ & $\begin{array}{c}\boldsymbol{I} \\
(\mathbf{N} \cdot \mathbf{s})\end{array}$ & $\begin{array}{c}\boldsymbol{V}_{\boldsymbol{0}} \\
(\mathbf{m} / \mathbf{s})\end{array}$ & $\boldsymbol{\varphi}_{\boldsymbol{q}}$ & $\boldsymbol{\Psi}$ & $\boldsymbol{\Pi}_{\boldsymbol{m a x}}$ & Damage mode \\
\hline A-22 & 14.60 & 56.51 & 8.47 & 6.78 & 0.0017 & 6.99 & Mode I-A \\
A-33 & 9.26 & 56.51 & 5.65 & 3.02 & 0.0011 & 3.04 & Mode I-A \\
A-34 & 10.82 & 56.51 & 5.65 & 3.02 & 0.0011 & 3.55 & Mode I-A \\
B-21 & 27.31 & 114.67 & 17.19 & 13.77 & 0.0034 & 13.72 & Mode I-A \\
B-22 & 26.67 & 114.67 & 17.19 & 13.77 & 0.0034 & 13.68 & Mode I-A \\
B-33 & 18.47 & 114.67 & 11.46 & 6.12 & 0.0023 & 6.46 & Mode I-A \\
C-21 & - & 175.94 & 26.37 & 21.12 & 0.0053 & 93.06 & Mode II \\
C-22 & - & 175.94 & 26.37 & 21.12 & 0.0053 & 243.33 & Mode II \\
C-33 & 95.35 & 175.94 & 17.58 & 9.38 & 0.0035 & 32.43 & Mode I-B \\
C-34 & 89.01 & 175.94 & 17.58 & 9.38 & 0.0035 & 29.77 & Mode I-B \\
\hline
\end{tabular}

\section{Conclusions}

Experimental investigation showed the blast behaviour of the clamped quadrangular aluminium plates in large scale, where special focus is placed on structural response and permanent deflection of the panel. It is shown that all plates subjected to free airblast loading in such conditions exhibited plastic permanent deformation with distinct counterintuitive behaviour. The damage modes were identified as Mode I and II, herein, Mode I could be subdivided into Mode I-A and Mode I-B.

For the dimensionless numbers concerned, a map of the counterintuitive region defined by $\Psi$ is obtained but not limit for $0.0011<\Psi<0.0053$, it also implies that $\Psi$ of 0.0034 is a critical value to distinguish the subdivision of Mode I. Moreover, $\varphi_{q}$ is lack in regularity to describe the damage mode but appears to be a significant indicator to predict permanent deflection when the plate deforms as Mode I-A. It should be noted that the results has examined the blast behaviour in large scale, notwithstanding its limitation, these observations do suggest the application of non-dimensional numbers in estimating the permanent damage of clamped aluminium plates.

The authors are grateful to the support of Innovative group of material and structure impact dynamics (No.11521062) and the Fundamental Research Project (No.2016602B003). 


\section{References}

1. T. Børvik, A. G. Hanssen, S. Dey, H. Langberg and M. Langseth, Eng. Stru. 30, 1605-1620 (2008)

2. G.N. Nurick and J.B. Martin, Int. J. Imp. Eng. 8, 15969 (1989)

3. G.N. Nurick and J.B. Martin, Int. J. Imp. Eng. 8, 171186 (1989)

4. G.N. Nurick and G.C. Shave, Int. J. Imp. Eng. 18, 99116 (1996)

5. S. Menkes and H. Opat, Exp. Mech. 13, 480-6 (1973)

6. G.S. Langdon, W.C. Lee and L.A. Louca, Int. J. Imp. Eng. 78, 150-160 (2015)

7. V. Aune, E. Fagerholt, K.O. Hauge, M. Langseth and T. Børvik, Int. J. Imp. Eng. 90, 106-121 (2016)

8. T.X. Yu, structural Crashworthiness and failure, 341384 (1993)
9. Q.M. Li, L.M. Zhao and G.T. Yang, Int. J. Imp. Eng. 11, 341-348 (1991)

10. E.A. Flores-Johnson, and Q.M. Li, Int. J. Imp. Eng. 38, 136-138 (2010)

11. V. Aune, G. Valsamos, F. Casadei, M. Larcher, M. Langseth and T. Børvik, Int. J. Imp. Eng. 99, 131-144 (2017)

12. R.J. Curry and G.S. Langdon, Int. J. Imp. Eng. 102, 102-116 (2016)

13. Q.M. Li, and N. Jones, Arch. Appl. Mech. 70, 245-254 (2000)

14. Y.P. Zhao, Arch. Appl. Mech. 68, 524-538 (1998)

15. N. Jacob, S.C.K. Yuen, G.N. Nurick, D.Bonorchis, S.A. Desai, D. Tait, Int. J. Imp. Eng. 30, 1179-1208 (2004) 